\title{
SINGULIÈRE MOBILISATION, LE RÉSEAU ÉDUCATION SANS FRONTIÈRES
}

\author{
Claudette Lafaye et Damien de Blic
}

C.E.R.A.S | Revue Projet

$2011 / 2-n^{\circ} 321$

pages 12 à 19

ISSN 0033-0884

Article disponible en ligne à l'adresse:

http://www.cairn.info/revue-projet-2011-2-page-12.htm

Pour citer cet article :

Lafaye Claudette et de Blic Damien, « Singulière mobilisation, le Réseau éducation sans frontières », Revue Projet, 2011/2 n³21, p. 12-19. DOI : 10.3917/pro.321.0012

Distribution électronique Cairn.info pour C.E.R.A.S.

(c) C.E.R.A.S. Tous droits réservés pour tous pays.

La reproduction ou représentation de cet article, notamment par photocopie, n'est autorisée que dans les limites des conditions générales d'utilisation du site ou, le cas échéant, des conditions générales de la licence souscrite par votre établissement. Toute autre reproduction ou représentation, en tout ou partie, sous quelque forme et de quelque manière que ce soit, est interdite sauf accord préalable et écrit de l'éditeur, en dehors des cas prévus par la législation en vigueur en France. II est précisé que son stockage dans une base de données est également interdit. 
Damien de Blic et Claudette Lafaye

Alors que les politiques migratoires se durcissent, des parents découvrent, à travers l'école de leurs enfants, des familles sanspapiers menacées $d^{\prime}$ expulsion. Le Réseau éducation sans frontières leur permet une mobilisation originale.

e Réseau éducation sans frontières (RESF) a fortement contribué à redonner une visibilité à la question des sans-papiers depuis le milieu des années 2000. Confrontés à la menace d'expulsion d'enfants scolarisés en France et nés de parents en situation irrégulière, des citoyens ont su enclencher une dynamique de contestation originale.

\section{- Dures politiques migratoires}

La question des mobilisations de et autour des sans-papiers s'inscrit dans un double contexte de transformation des formes et de la structure de l'immigration, depuis les années 1970, et d'une série de lois pour la contrôler et la limiter. Dans l'après-guerre, l'économie française avait besoin de main-d'œuvre. Une population croissante d'étrangers entre alors en France, avec un passeport de tourisme, ou clandestinement. Sans peine, elle trouve à s'embaucher et obtient, a posteriori, la carte de travail et la carte de séjour permettant de régulariser sa situation. Mais les années 1970

Damien de Blic est maître de conférences en science politique à l'Université Paris 8.

Claudette Lafaye est maître de conférences en sociologie à l'Université Paris 8. 
marquent un terme durable à ce mouvement, amorçant une politique de fermeture des frontières vis-à-vis de l'immigration de travail. La réglementation accompagne les évolutions structurelles de la population immigrée, qui passe d'une main d'œuvre essentiellement masculine appelée à repartir, à une population familiale qui va demeurer sur le territoire. En 1976, un décret instaure pour la première fois, sous conditions de ressources et de logement, le regroupement familial. L’ancienneté du séjour en France, les attaches personnelles et familiales deviennent prépondérantes sur le travail pour donner accès au séjour. Si l'arsenal juridique se fait toujours plus restrictif à l'encontre de l'immigration - notamment irrégulière -, la gauche, durant les trois alternances, préserve la valeur de l'ancienneté du séjour et des attaches personnelles et familiales en France. La régularisation de 130000 étrangers réalisée en 1981 par le gouvernement Mauroy est encore axée sur le travail, mais en 1997, la circulaire Chevènement (80 000 personnes régularisées) privilégie clairement les familles au détriment des célibataires sans attaches.

La période ouverte en 2002 est marquée par une inflation de textes législatifs, qui restreignent délibérément les possibilités de séjour régulier pour les étrangers et durcissent considérablement les politiques publiques à destination des immigrés en situation irrégulière : trois lois et une ordonnance sont promulguées entre 2003 et 2007 et pas moins de 29 décrets d'application entre 2006 et 2008². En ce début d'année 2011, une nouvelle loi est en discussion au Parlement. Le séjour des étrangers se trouve toujours plus encadré et les possibilités de régularisation toujours plus réduites. La politique d'immigration s'inscrit, dès le milieu des années 2000, dans un renouveau de la promotion de l'immigration économique, avec l'élaboration des catégories d'immigration «choisie " et d'immigration "subie ». Et depuis 2003, le ministère de l'Intérieur ${ }^{2}$ fixe des objectifs chiffrés en matière de reconduites à la frontière. Cette volonté politique semble porter ses fruits, comme en témoignent les chiffres annoncés à la fin de chaque année civile : pour 2009, 29000 reconduites à la frontière auraient été réalisées. C'est dans ce contexte que des familles et des jeunes majeurs, relativement protégés jusque-là, se retrouvent sous la menace d'une expulsion.

1. Cf. Groupe d'information et de soutien des immigrés (Gisti), Guide de l'entrée et du séjour des étrangers en France, La Découverte, 2009.

2. Entre mai 2007 et novembre 2010, la politique en direction des migrants et des étrangers a été regroupée au sein d'un ministère de l'Immigration, de l'Intégration, de l'Identité nationale et du Développement solidaire. 
Ce qui va devenir le collectif RESF naît en 2004, à l'initiative d'enseignants d'un lycée d'enseignement professionnel d'Île-de-France, confrontés au cas d'élèves qui se trouvent du jour au lendemain, à leur majorité, en situation irrégulière. Il s'ensuit un appel en faveur d'une mobilisation pour la régularisation des centaines, voire des milliers d'autres jeunes dans la même situation. Cet appel trouve une nouvelle résonnance à l'automne 2005, lorsque des mineurs sont menacés d'expulsion après la mise en rétention ou un arrêté préfectoral de reconduite reçu par leurs parents. Le gouvernement accorde un "sursis » aux familles concernées jusqu'à la fin de l'année scolaire. L'approche de cette échéance et la découverte, dans de très nombreuses écoles maternelles et élémentaires, mais aussi dans des collèges, de cas similaires suscitent la création de multiples comités locaux de soutien à ces enfants et à leurs parents. Ces comités se coordonnent au sein du Réseau éducation sans frontières ${ }^{3}$.

\section{- Découvrir son voisin comme sans-papiers}

Constitués de parents d'élèves, d'enseignants, de personnels d'établissements, parfois de voisins, les comités se donnent pour objectifs la régularisation des jeunes majeurs et des familles sans-papiers qui se sont révélés à eux et la protection contre l'expulsion de ceux qui n'ont pu obtenir cette régularisation. Apparaît ainsi l'un des traits distinctifs de cette action collective : ce ne sont pas ici les sans-papiers eux-mêmes qui sont mobilisés, ni les soutiens traditionnels (les syndicats et les Églises) ${ }^{4}$, mais des personnes ordinaires, le plus souvent des parents d'élèves.

L'engagement dans RESF ne naît pas nécessairement d'une sensibilité préexistante à la cause des sans-papiers, mais de situations singulières qui réclament un engagement immédiat ${ }^{5}$. Devenir militant, c'est, dans bien des cas, éprouver le choc d'une découverte : que des sans-papiers puissent appartenir à son univers le plus quotidien, celui de l'école fréquentée par ses propres enfants. Lorsque des familles en situation irrégulière appro-

3. RESF se caractérise par une faible structuration et des contours mouvants : il n'y a pas d'adhésion formalisée, ni de paiement d'une cotisation, comme cela est généralement le cas dans les associations loi 1901. Les comités locaux, départementaux et le comité national ne disposent pas de présidents élus ou de représentants mandatés, mais seulement de porte-parole. La coordination est réalisée essentiellement au travers de listes de messagerie électronique à diffusion plus ou moins élargie. Cela entraîne une impossibilité de dénombrer les militants.

4. Voir Johanna Siméant, La cause des sans-papiers, Presses de Sciences Po, 1998.

5. Nous nous appuyons sur une enquête que nous menons actuellement dans le cadre du Groupe de sociologie politique et morale (GSPM, CNRS-EHESS), centrée sur l'émergence et le développement de RESF à Paris. 
chent les parents d'élèves, ceux par exemple des conseils d'école, ces derniers découvrent que les sans-papiers, souvent associés jusqu'alors au monde lointain de la clandestinité, aux marges les plus obscures du travail, voire à la " jungle " de Sangatte, constituent en fait une réalité présente, à portée de main et de regard. Interpellés par des familles qui demandent aide et soutien, pour répondre à un courrier préfectoral lourd d'une menace d'expulsion ou pour constituer un dossier de demande de régularisation, les parents d'élèves éprouvent une responsabilité nouvelle. Cette découverte de la proximité s'accompagne d'une autre : celle de la violence exercée par l'État, dans le geste même de l'expulsion, ou sa menace, et dans la disproportion ressentie entre le motif de l'action de l'État (l'irrégularité administrative) et la brutalité de la sanction encourue (l'arrestation, l'emprisonnement dans un centre de rétention administrative, les menottes aux poignets à l'arrivée au tribunal).

Le répertoire d'action développé par RESF ne saurait être compris sans tenir compte de sa naissance au sein de l'univers scolaire. L'école a rendu possible ce mouvement de soutien de Français ordinaires aux sanspapiers : elle est un des rares lieux de rencontre entre les uns et les autres. Du fait de leur appartenance à la communauté scolaire, les parents sans papiers ne sont pas identifiés immédiatement comme tels; ils sont avant tout les parents de camarades de classe de leurs propres enfants. L'école offre enfin une justification publique exprimable en termes de droits : le droit à l'éducation. Lorsque des enfants sont renvoyés dans un pays où ils n'ont parfois jamais vécu, loin des attaches qu'ils ont pu construire en France, leur scolarité est brutalement interrompue, leur droit à l'éducation nié. De plus, si l'école mobilise les valeurs de la solidarité républicaine, elle affilie aussi à une communauté de voisinage, sur la base de la sectorisation géographique.

\section{-Appartenir à une communauté de voisinage}

Les militants de RESF demandent que soit reconnue l'appartenance des familles sans papiers à ces communautés dont ils sont membres à part entière. Cette revendication d'appartenance court à travers les slogans volontiers mobilisés par le réseau : "Ils sont ici, parmi nous ", "Ils paient des impôts ", "Ils enrichissent la France ", "Leurs enfants sont nés en France ", "Leurs enfants sont les copains de nos enfants", "Ils sont nos voisins ». Une appartenance que les actions concrètes développées par les comités RESF s'efforcent de mettre en pleine lumière. 
La solidarité de la communauté scolaire ou du voisinage s'exprime depuis les situations les plus dramatiques, réclamant une intervention d'urgence, jusqu'à la défense au long cours des dossiers de demande de régularisation. Quelle que soit la forme prise (rassemblement devant le commissariat, appels téléphoniques, envoi d'emails et de fax au commissariat, à la préfecture et au ministère, présence physique sur les bancs des tribunaux, journée "école morte "... ${ }^{6}$ ), il s'agit de manifester aux autorités que la personne sans papiers n'est pas une personne isolée, qu'elle est membre d'une communauté de proximité, qui dépasse l'affiliation communautaire propre au pays d'origine, et de rendre celle-ci visible.

Une autre forme d'action se traduit par le maillage, plus ou moins dense selon les contextes, créé par les comités locaux autour des jeunes majeurs ou des familles. Ceux-ci seront aidés dans la constitution de leur dossier de demande de régularisation. Ils seront accompagnés, lors des convocations à la préfecture. Au-delà d'un rôle de traduction ou de médiation, les accompagnateurs contribuent, par leur seule présence, à faire monter l'exigence de publicité et à installer un cadre qui assure aux personnes sans papiers qu'elles ne seront pas maltraitées. Cette insertion dans un réseau de liens modifie radicalement leur place. Non seulement elles ont cessé d'être isolées et invisibles, mais ceux avec lesquels elles entrent en interaction se sont multipliés. En retour, ce maillage autour des sanspapiers a pour effet de tisser et d'intensifier les liens entre l'ensemble des habitants engagés, qui sont conduits à multiplier leurs communications et leurs échanges.

Le parrainage républicain permet à une personne ou famille sanspapiers et à celles et ceux engagés auprès d'eux d'exprimer publiquement leurs attaches et leur solidarité. Les parrainages collectifs sont un legs des mobilisations de 1997-1998, notamment après la régularisation partielle opérée par la circulaire Chevènement: plusieurs dizaines de milliers de sans-papiers particulièrement vulnérables avaient été déboutés. Aujourd'hui, à Paris surtout, ces parrainages ont systématiquement lieu dans le cadre des mairies d'arrondissement, réinvesties comme "maisons communes ». Ils allient la solennité du cadre républicain, renforcée par la présence systématique du maire et de nombreux élus, tout en inscrivant le geste de solidarité dans un espace de proximité qui fait sens pour les acteurs.

6. L'entretien avec Brigitte Wieser, pp. 4-11 de ce numéro, évoque quelques-uns de ces modes d'action. 


\section{Donner un visage aux chiffres}

Ces exemples dessinent une mobilisation fondée sur des attaches localisées, où la singularité des liens tissés entre Français et sans-papiers est valorisée. Faut-il en conclure que RESF pratique le cas par cas? La réponse est négative pour au moins trois raisons.

La première tient à la place occupée par la communauté d'appartenance sur laquelle, comme on vient de le voir, insistent les protestations. Ce n'est pas seulement une personne ou une famille qui est affectée par l'expulsion ou la menace d'expulsion, mais une communauté dans son ensemble. Le problème n'est pas uniquement celui de la famille sans papiers, il est celui du milieu d'interconnaissance, des voisins, des parents, des enseignants qui se sentent personnellement éprouvés.

La deuxième raison est que l'insistance sur le caractère personnel de la solidarité exprimée a un sens politique. Ces gestes de solidarité peuvent être lus comme une réponse en acte à l'anonymat du chiffre des expulsions. "Les chiffres ont un visage ", slogan employé dans plusieurs circonstances dans le réseau, témoigne du souci de ne pas déshumaniser la cause des sans-papiers et joue comme un rappel à vocation critique : ne pas oublier, derrière les chiffres préfectoraux (de refoulés, de déboutés, d'expulsés, etc.), la souffrance, bien réelle, qu'elle tend à abstraire. Derrière la cause générale des sans-papiers, on risque d'oublier sa responsabilité individuelle vis-à-vis des sans-papiers, êtres de chair et d'os.

Enfin, ces actions font l'objet d'une circulation et d'un cumul d'expérience au sein d'un réseau national. Ce réseau est porteur de revendications d'une portée plus générale que la défense de cas singuliers, puisque ceux qui y participent s'accordent sur la revendication d'une régularisation sur la base de la scolarisation. Le réseau peut être vu comme une forme de généralisation. La constitution même de RESF s'opère selon un processus de contagion, comme le décrit un de ses fondateurs, Pierre Cordelier, enseignant à la retraite : "La vie réelle $(. .$.$) - c'est une bonne nouvelle$ - est contagieuse. À chaque cas sortant de l'ombre, on a vu une mobilisation se produire. À partir de l'établissement scolaire, du quartier. Mais aussi à chaque cas révélé, d'autres ont suivi, comme si une porte s'était ouverte pour enfin sortir de la solitude cachée (...) et la contagion s'étend aussi aux familles. Des parents sans papiers de jeunes enfants scolarisés nous trouvent. Des enseignants aussi qui ne savent que faire. Et des voisins (...). Le réseau est là : les comités locaux se multiplient, écoles, quartiers, 
villages, villes, départements. Toujours à chaque fois, sur une vie, sur une famille. " ${ }^{7}$ Par son ampleur, le réseau est la preuve que le mal qui frappe les sans-papiers et les communautés auxquelles ils appartiennent est général. Il témoigne aussi d'une capacité collective à réagir et à y faire face.

\section{- Renouveler la critique sociale}

Au sein des mobilisations autour des sans-papiers, RESF occupe une place singulière. Ses différentes mobilisations s'inscrivent dans des dynamiques et des histoires spécifiques : leur horizon comme leur forme s'élaborent et se reconfigurent continûment dans les interactions et les confrontations avec les politiques publiques à destination des migrants. C'est ainsi que le renouveau de la promotion de l'immigration économique s'accompagne depuis trois ans, en France, d'une réactivation de la figure du travailleur sans-papiers, revendiquant une régularisation associée à la grève du travail $^{8}$. En Italie, le débat public et la contestation se sont focalisés, au milieu des années 2000, sur la question de la rétention et de l'enfermement des étrangers ${ }^{9}$, éléments largement ignorés en France au même moment. Les formes même des mobilisations s'inscrivent dans des histoires contrastées et parfois croisées. Aux États-Unis, les sans-papiers latino-américains qui défilent au printemps 2006, ne s'appuient pas sur les traditions américaines de mobilisation fondée sur la communauté, mais ils importent la manifestation de masse dans les rues de $\mathrm{Chicago}^{10}$.

À l'instar des précédentes mobilisations autour des sans-papiers, ou des mobilisations de chômeurs ou de mal logés, RESF participe du renouveau des formes de la critique sociale en France : il s'agit moins de conquérir le pouvoir que de chercher à faire accéder les conflits sociaux à une expression publique. Peut-on parler pour autant d'une nouvelle forme de militantisme? Jacques Ion a défendu la thèse d'une mutation des formes de l'engagement ${ }^{11}$. L'engagement classique (syndical, partisan ou associatif), caractérisé par l'emprise du groupe sur l'individu, par une soumission de

7. Avant-propos dans Miguel Benasayag, Angélique del Rey et des militants de RESF, La chasse aux enfants, La Découverte, 2008.

8. Cf. Asplan, "Travailleurs sans papiers : la précarité interdite ", Les mondes du travail, n 7, 2009; Pierre Barron, Anne Bory, Sébastien Chauvin, Nicolas Jounin, Lucie Tourette, "L'intérim en grève : la mobilisation des travailleurs sans papiers intérimaires ", Savoir/Agir, n 12, 2010.

9. Voir Bruno Cousin et Tommaso Vitale, «La gauche italienne face au mouvement pour les libertés civiles des sans-papiers ", Critique internationale, $\mathrm{n}^{\circ} 37$, octobre-décembre 2007.

10. Voir Mathieu Bonzom et Sébastien Chauvin, "Les sans-papiers dans la rue ", La vie des idées,

18 août 2007, www.laviedesidees.fr/Les-sans-papiers-dans-la-rue.html.

11. Jacques Ion, La fin des militants?, L'Atelier, 1997. 
celui-ci à la hiérarchie militante, par l'importance des rites d'entrée et une forte dimension identitaire, aurait cédé la place, en différents lieux, à un engagement distancié, plus fluctuant, marqué par un affranchissement des appartenances collectives. Cette thèse a été contestée sur plusieurs fronts : contribution à la disqualification des pratiques politiques des classes populaires, existence d'engagements fluides dans le passé, intensité de certains engagements contemporains, maintien de formes bureaucratiques et hiérarchiques au sein de mouvements caractéristiques du militantisme en réseau ${ }^{12}$. En faisant feu de tout bois, cette critique finit par perdre de vue la portée heuristique des deux figures de l'engagement dégagées par Jacques Ion.

Sur de nombreux aspects, RESF s'affranchit du modèle de l'engagement militant classique : ce n'est pas une association, il n'y a pas d'adhérent, la participation peut y être intense ou ponctuelle, des collectifs locaux se mettent en sommeil puis se réactivent, à la faveur d'une arrestation ou d'une menace d'expulsion... Son originalité n'est pas dans sa proximité avec la figure de l'engagement distancié. Elle réside plutôt dans le fait d'autoriser en son sein des engagements différenciés, tant au niveau individuel que collectif ${ }^{13}$.

Damien de Blic et Claudette Lafaye

12. Lilian Mathieu, « Un 'nouveau militantisme’ ? À propos de quelques idées reçues », Contretemps, www.contretemps.eu/socio-flashs/nouveau-militantisme-propos-quelques-idees-recues.

13. L'entretien avec Brigitte Wieser, pp. 4-11 de ce numéro, souligne cet aspect. 